\title{
Neuron Model Interpretation of a Cyclic Motion Control Concept
}

\author{
Dominic Lakatos and Alin Albu-Schäffer
}

\begin{abstract}
Elastic properties of muscles and tendons are assumed to play a central role for the energy efficiency and robustness of locomotion in biological systems. Yet, the way in which the nervous system controls highly nonlinear body dynamics to produce stable periodic motions is far from being well understood. On the basis of a simple but very effective control law, which we developed and verified for variable impedance robots, we propose a controller model, which might be a very plausible hypothesis also for biological systems. The original robot controller has a bang-bang action triggered by the generalized force acting along a coordinate corresponding to the principal oscillation mode of the system. This coordinate is computed in a model-free, adaptive manner. It turns out that the control law can be easily realized with a neural network, whose weights are adapted according to the Hebbian learning rule. If this hypothesis is confirmed, cyclic body motions can be very easily and robustly implemented, with a surprisingly small number of neurons.
\end{abstract}

\section{INTRODUCTION}

Recently, bio-inspired robot designs and control methods [1], [2], [3], [4], [5], [6], [7], [8], [9] for the execution of explosive and cyclic motions have been proposed. The robot design concepts are based on the physical property of having elasticities between the actuators and the outputs of the joints, and are strongly inspired by the principle of skeletal muscles [10], [11]. The springs can be exploited to robustly handle mechanical impacts and to improve performance and energetic efficiency during periodic and explosive movement tasks. Especially in the case of cyclic motions such as walking, crawling, or jumping, and running, the elastic energy can be buffered and released to approach a resonance like behavior, where the effort to sustain the oscillation is minimal. Therefore, compliant actuators implement the technical concept of intrinsic mechanical oscillation modes and correspond with the active principle of biological actuators.

This paper investigates cyclic motion control principles, which are mainly intended for legged systems. Well established locomotion control concept are based on the spring loaded inverted pendulum (SLIP) model [12], [13], [14], [15]. The idea of the SLIP model is inspired by experimental observations of biologists [16] who hypothesize that highdimensional, nonlinear system dynamics anchored in a complex animal collapse to this simple template dynamics. Further related control methods are robotic implementations of central pattern generation (CPG) [17] and adaptive frequency oscillators (AFO) [18]. These approaches are inspired by observations in neuro-control units of amphibians, where a central unit composed of multiple, phase coupled oscillators,

The authors are with the Institute of Robotics and Mechatronics, German Aerospace Center (DLR), D-82234 Oberpfaffenhofen, Germany. Contact: dominic.lakatosedr.de generates complex multi degree-of-freedom (DOF) motions. Whereas the AFO approach of [18] considers feedback of the plant in the motion pattern generation, the CPGs and most of the SLIP based concepts are basically applied as open loop control.

In our recent papers [19], [20], [21] we introduced a control method which directly excites the intrinsic oscillatory dynamics of compliantly actuated robotic plants in a feedback manner. The controller comprises two main parts: a switching law to generate a limit cyclic along a single coordinate direction, and an adaptation law which converges to a transformation corresponding to the dominant oscillation mode of the plant. The limit cycle controller has been derived from observations of human motor control [19], [21]. It switches the (generalized) equilibrium position of a spring triggered by measurements of the (generalized) spring-force/deflection. The adaptive part recursively extracts the principal components of the joint motion and thereby accounts for the distribution of the excitation amplitudes (similar to the eigenvectors in linear oscillation theory) such that they fit to and properly excite the dominant intrinsic oscillation mode of the mechanical system [20]. In contrast to the template dynamics [12], [13] or CPG [17], [18], [15] based approaches, which address rigid actuators or full rigid body motions, our new cyclic motion control concept directly uses the oscillatory plant dynamics itself as oscillation unit. Therefore, this feedback controller has rapid convergence properties-mimicking the fast adaptive nature of biological systems.

The contribution of this paper is a biological neuron model interpretation of this new cyclic motion control concept. On the basis of correlation-based learning in a firing rate formalism [22, Chapt. 10.2], we interpret the controller as a biological neural network. Thereby, the switching controller represents a single neuron in the hidden layer of the network, which is driven by sensory neurons of the input layer via synapses and acts on the actuator neurons of the output layer via a synapses arrangement mirrored from the input layer. The adaptation of the synaptic efficacies is explained using the principle of Hebbian plasticity [23] and competition between synaptic weights [24]. Therefore, this neuron model turns out to be a biologically plausible representation of our cyclic motion control concept. Together with technically advantageous aspects such as robustness and energetic efficiency, the neuron model interpretation supports our hypothesis that the control principle might occur in biological systems in a similar form.

The paper is organized as follows: In the next section we describe the principle of the robotic control concept. 


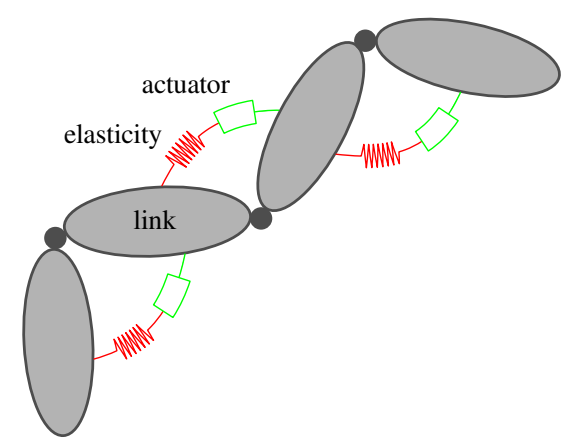

Fig. 1. Generic model of a compliantly actuated mechanical systems. In case of robotic systems the motor (actuator) acts via a mechanical spring (elasticity) on the preceding link. In case of a biological system such as human or animal limbs the muscles (actuators) act via tendons (elasticities) on the preceding links.

Then, in Sect. III we propose a neuron model interpretation of this controller. Sect. IV evaluates the performance of the controller in simulation. Finally, Sect. IV discusses and concludes the work.

\section{ROBOTIC CONTROL CONCEPT}

In this section, we introduce a generic model (Fig. 1) which represents bio-inspired, compliantly actuated robotic systems and can be straightforwardly modified to describe the biological counterparts with skeletal muscles as actuators. Then, on the basis of this model, we explain our cyclic motion control concept, as introduced in [20].

\section{A. Modeling compliantly actuated mechanical systems}

Compliantly actuated mechanical systems can be represented by Euler-Lagrange equations [25], satisfying

$$
\frac{\mathrm{d}}{\mathrm{d} t}\left(\frac{\partial L(\boldsymbol{x}, \dot{\boldsymbol{x}})}{\partial \dot{\boldsymbol{x}}}\right)-\frac{\partial L(\boldsymbol{x}, \dot{\boldsymbol{x}})}{\partial \boldsymbol{x}}=\boldsymbol{\tau}_{\text {gen }}-\boldsymbol{d}(\boldsymbol{x}, \dot{\boldsymbol{x}}),
$$

where the Lagrangian $L(\boldsymbol{x}, \dot{\boldsymbol{x}})=T(\boldsymbol{x}, \dot{\boldsymbol{x}})-U(\boldsymbol{x})$ is the difference of the kinetic energy $T(\boldsymbol{x}, \dot{\boldsymbol{x}})$ and potential energy $U(\boldsymbol{x})$. The potential energy $U(\boldsymbol{x})=U_{g}(\boldsymbol{x})+U_{\psi}(\boldsymbol{x})$ is the sum of a gravity and elastic potential, respectively. The vector of coordinate $\boldsymbol{x}=\left(\boldsymbol{\theta}^{T}, \boldsymbol{q}^{T}\right)^{T} \in \mathbb{R}^{m+n}$ can be divided into actuator coordinates $\boldsymbol{\theta} \in \mathbb{R}^{m}$ and link coordinates $\boldsymbol{q} \in \mathbb{R}^{n}$. The vector of generalized forces $\boldsymbol{\tau}_{\text {gen }}=\left(\boldsymbol{u}^{T}, \boldsymbol{\tau}_{\text {ext }}^{T}\right)^{T}$ is composed of the control input $\boldsymbol{u} \in \mathbb{R}^{m}$ and the external torques $\boldsymbol{\tau}_{\text {ext }} \in \mathbb{R}^{n}$. Moreover, the term $\boldsymbol{d}(\boldsymbol{x}, \dot{\boldsymbol{x}}) \in \mathbb{R}^{m+n}$ represents a dissipative force, where $\dot{\boldsymbol{x}}^{T} \boldsymbol{d}(\boldsymbol{x}, \dot{\boldsymbol{x}}) \geq 0$ holds.

In case of robotic systems, $\left(\boldsymbol{\theta}^{T}, \dot{\boldsymbol{\theta}}^{T}\right)^{T}$ represent the states of the motor dynamics. They are directly actuated via the motor torque $\tau_{\mathrm{m}}:=\boldsymbol{u}$. In case of biomechanical systems, $\left(\boldsymbol{\theta}^{T}, \dot{\boldsymbol{\theta}}^{T}\right)^{T}$ could represent the internal states of second order muscle dynamics. Thereby, for instance, the muscle activation is the control input, 1.e. $\boldsymbol{a}:=\boldsymbol{u}$.

For the explanation of the robotic controller given next, we consider the motor PD control

$$
\boldsymbol{u}=-\boldsymbol{K}_{\mathrm{D}} \dot{\boldsymbol{\theta}}-\boldsymbol{K}_{\mathrm{P}}\left(\boldsymbol{\theta}-\boldsymbol{\theta}_{\mathrm{d}}\right),
$$

where $\boldsymbol{K}_{\mathrm{D}}, \boldsymbol{K}_{\mathrm{P}} \in \mathbb{R}^{m \times m}$ are symmetric and positive definite controller gain matrices, and $\boldsymbol{\theta}_{\mathrm{d}} \in \mathbb{R}^{m}$ is the desired motor position, for the system (1). Then, using classical assumptions for robotic systems (high controller gains and singular perturbation [26]), 1.e. $\boldsymbol{\theta} \approx \boldsymbol{\theta}_{\mathrm{d}}$, we consider the motor position $\boldsymbol{\theta}$ as control input.

\section{B. Cyclic motion control concept}

To excite and sustain a cyclic motion along an intrinsic mechanical oscillation mode of the considered system, two technical aspects are of major importance:

First, the timing of the energy input has to be controlled. In the ideal case the frequency of the energy input is close to the "natural" frequency of the oscillation mode such that the system oscillates in resonance. Technically, this is achieved using the switching based control law

$$
\theta_{z}\left(\varphi_{z}\right)=\left\{\begin{array}{cc}
\operatorname{sign}\left(\varphi_{z}\right) \hat{\theta}_{z} & \text { if }\left|\varphi_{z}\right|>\epsilon_{\varphi_{z}} \\
0 & \text { otherwise }
\end{array}\right.
$$

Herein,

$$
\varphi_{z}=\boldsymbol{w}^{T}(\boldsymbol{\theta}-\boldsymbol{q}) \in \mathbb{R}
$$

is the generalized spring deflection of the oscillation mode ${ }^{1}$, $\epsilon_{\varphi_{z}} \in \mathbb{R}_{>0}$ the corresponding threshold and $\hat{\theta}_{z} \in \mathbb{R}$ the modal switching amplitude.

Second, the direction of the "step-like" excitation has to be adapted to coincide with the (local) direction of the oscillation mode [20]. This is achieved using the adaptation dynamics

$$
\dot{\boldsymbol{w}}(t)=\gamma z(t)(\boldsymbol{q}(t)-z(t) \boldsymbol{w}(t)),
$$

where $z(t)=\boldsymbol{w}(t)^{T} \boldsymbol{q}(t)$ is a local approximation of the modal coordinate and $\gamma \ll 1$ is an adaptation gain. The adaptation law (5) extracts the basis vector $\boldsymbol{w} \in \mathbb{R}^{m}$ corresponding to the major principal component of the joint motion $\boldsymbol{q}(t)$ [27] and therefore the direction of the oscillation mode [28]. If the adaptation dynamics (5) converges sufficiently fast, a "step-like" excitation in the required direction is approached by

$$
\boldsymbol{\theta}=\boldsymbol{\theta}_{0}+\boldsymbol{w} \theta_{z}
$$

\footnotetext{
${ }^{1}$ The generalized spring deflection performs work at the joint elasticities along the oscillation mode.
}

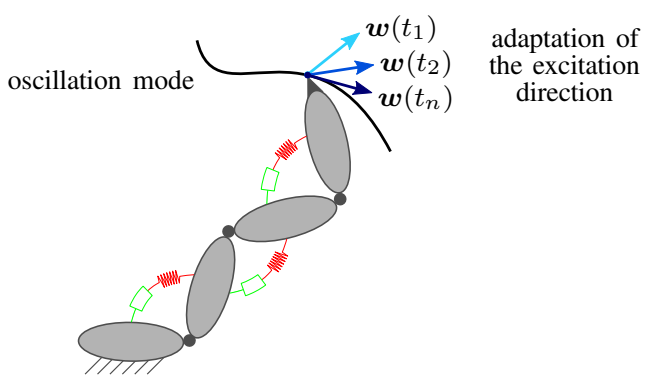

Fig. 2. Principle of the cyclic motion controller. The step-by-step convergence of the excitation to the oscillation mode is shown. 
In summary, the controller principle can be explained as follows (Fig. 2): Initially, the system is excited with a first guess of the modal direction $\boldsymbol{w}\left(t_{1}\right)$ at time instance $t_{1}$. Then, the resulting oscillatory motion is observed (cf. (5)). When the generalized spring deflection reaches the threshold value (cf. (3)), the system is excited again with an improved guess of the modal direction $\boldsymbol{w}\left(t_{2}\right)$. By repeating this procedure, the oscillation reaches a steady-state, which is close to the intrinsic mechanical oscillation mode of the system.

Remark 1: In the derivation of the cyclic motion controller (3)-(6) we have assumed that $\boldsymbol{\theta}_{0}=\mathbf{0}$ is the center of the oscillation. The extension for a general center of the oscillation is given in the Appendix I.

Remark 2: Since the controller changes the intrinsic dynamics properties of the plant only to a minimum extent, the plant itself must have damping properties such that oscillation excited in undesired modes decay faster than in the desired mode. However, serially structured elastic multibody systems such as robotic and human arms and legs display these beneficial damping properties [21].

Remark 3: Note that the controller requires no model knowledge of the plant. Only measurements of the states at position level are required. Therefore, the approach is very robust (see, [20] and the appended video ${ }^{2}$ ).

\section{NEURON MODEL}

Besides the technical advantages discussed in the previous section, a very promising property of the cyclic motion control concept is its surprisingly simple interpretation as a biological neural network. The general idea of this biologically plausible neural control is shown in Fig. 3. The sensory signals corresponding to the deflections of the joint elasticities (measured in biological systems by the Golgi organs) act on the input neurons of the network. These signals are transmitted via synapses with efficacies $w_{i}$ and summed up at a single neuron in the hidden layer. The cumulative

${ }^{2}$ Video link: www.in.tum.de/index.php?id=6123

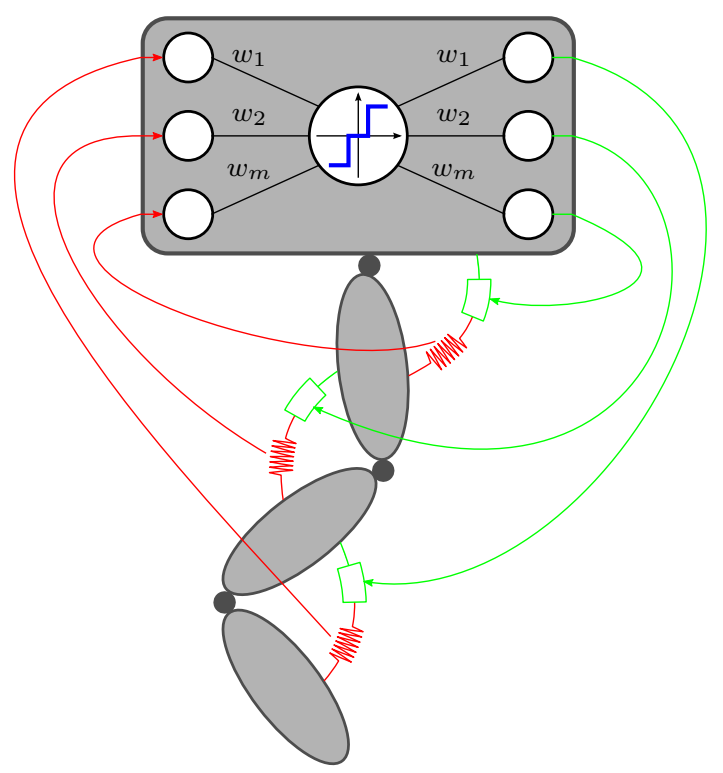

Fig. 3. Neuron model interpretation of the cyclic motion controller. signal is then processed in this neuron using a classical threshold function of a firing rate model and distributed over the output neurons of the network via additional synapses. The output neurons encode desired equilibrium positions, more precisely, step changes of the desired positions, for each muscle, according to the equilibrium point hypothesis [29]. In the following we explain how the principle of the switching controller and the adaptation law coincide with well established models of the biological counterparts.

\section{A. Switching behavior of firing rate coded neuron models}

The main principle of the switching controller presented in Sect. II is similar to the simplest form of a firing rate coded neuron model [30]:

$$
y=\phi\left(\nu-\epsilon_{\nu}\right) \text {. }
$$

Herein $\nu$ denotes the input firing rate and $\phi$ is a threshold function. The input firing rate is scaled and pushed through a nonlinear function. In more detail, if the value of the input signal exceeds the limit $\epsilon_{\nu}$, the output is changed step wise by a predefined amount. This step wise change maintains as long the input undercuts the threshold implication. Note that if $\epsilon_{\nu}=0$, this neuron model coincides with the switching control (3) introduced in Sect. II. A switching behavior corresponding to $\epsilon_{\phi_{z}}>0$ can be achieved by combining multiple neurons with $\epsilon_{\nu} \neq 0$.

\section{B. Adaptation of synaptic efficacies}

The adaptation of the synaptic efficacies will be explained in a correlation-based firing rate formalism [22, Chapt. 10.2]. Thereby, the two basic principles, Hebbian plasticity [23] and competition between synaptic weights [24], will be consulted.

To this end, let us consider a single synapse with efficacy $w_{i}$ connecting a pre- and postsynaptic neuron of the input and hidden layer, respectively. Then, for this single synapse, we consider the prototype of Hebbian learning,

$$
\frac{\mathrm{d}}{\mathrm{d} t} w_{i}=c_{2}^{\mathrm{corr}} \nu_{i}^{\mathrm{pre}} \nu^{\mathrm{post}}
$$

where $\nu_{i}^{\text {pre }}$ and $\nu^{\text {post }}$ represents the firing rate of the preand postsynaptic neuron, respectively, and $c_{2}^{\text {corr }}$ is a positive constant. The learning rule (8) accounts for the two particularly important aspects in Hebb's postulate: locality, 1.e. the change of the synaptic efficacy can depend only on local variables (pre- and postsynaptic firing rate), and cooperativity, 1.e. pre- and postsynaptic neurons have to fire simultaneously to change the synaptic efficacy. Now, let us consider an ensemble of $N$ patterns $\left\{\boldsymbol{\xi}\left(t_{1}\right), \ldots, \boldsymbol{\xi}\left(t_{N}\right)\right\}$ where at each time step $t_{j}$ one of the patterns $\boldsymbol{\xi}\left(t_{j}\right)$ is fixing the firing rate of the presynaptic neurons, 1.e., $\nu_{i}^{\text {pre }}\left(t_{j}\right)=$ $\xi_{i}\left(t_{j}\right)$. Assuming that each pattern $\boldsymbol{\xi}\left(t_{j}\right)$ is presented for a short duration $\Delta t$ such that the weight changes by a small amount only, 1.e., $\int_{t}^{t+\Delta t} \frac{\mathrm{d} w_{i}(\tau)}{\mathrm{d} \tau} \mathrm{d} \tau \ll w_{j}(t)$, we can take the postsynaptic firing rate $\nu^{\text {post }}(t)=\boldsymbol{w}(t)^{T} \boldsymbol{\xi}(t)$ as constant for 
this duration. Therefore, the total weight change due to the presentation of $\boldsymbol{\xi}\left(t_{j}\right)$ (to first order in $\Delta t$ ) is

$$
\begin{aligned}
& \Delta \boldsymbol{w}(t)=\boldsymbol{w}(t+\Delta t)-\boldsymbol{w}(t)= \\
& \gamma\left(\boldsymbol{w}(t)^{T} \boldsymbol{\xi}(t)\right) \boldsymbol{\xi}(t)+\mathcal{O}\left(\Delta t^{2}\right),
\end{aligned}
$$

where $\gamma=c_{2}^{\text {corr }} \Delta t$.

The Hebbian learning rule in the simple form (8) leads to exponentially growing weights. Since, this is biologically not plausible, an additional aspect of learning has to be taken into account, viz. competition. That means a synaptic efficacy increases at the expense of other synaptic efficacies (acting on the same postsynaptic neuron) $)^{3}$. Competition of synaptic weights can be modeled mathematically by normalization of the weight vector to a constant length. In the following, we consider multiplicative normalization of $\|\boldsymbol{w}\|$ which in turn leads to Oja's learning rule [31]. Therefore, consider the weight change $\Delta \tilde{\boldsymbol{w}}(t)$ due to the simple Hebbian learning rule resulting from (9),

$$
\Delta \tilde{\boldsymbol{w}}(t)=\gamma\left(\boldsymbol{w}(t)^{T} \boldsymbol{\xi}(t)\right) \boldsymbol{\xi}(t) .
$$

Then, performing a normalization on the update of the weights (after $\Delta t$ ) to unity length, 1.e.,

$$
\boldsymbol{w}(t+\Delta t)=\frac{\boldsymbol{w}(t)+\Delta \tilde{\boldsymbol{w}}(t)}{\|\boldsymbol{w}(t)+\Delta \tilde{\boldsymbol{w}}(t)\|},
$$

and assuming again a small weight change during $\Delta t$ $(\gamma \ll 1)$, we obtain the weight change $\Delta \boldsymbol{w}(t)$ including normalization up to first order in $\gamma$ (Appendix II),

$$
\Delta \boldsymbol{w}(t)=\Delta \tilde{\boldsymbol{w}}(t)-\boldsymbol{w}(t)\left(\boldsymbol{w}(t)^{T} \Delta \tilde{\boldsymbol{w}}(t)\right)+\mathcal{O}\left(\gamma^{2}\right) .
$$

Dividing the above equation by $\Delta t$ and approximating the differences by differential operators, we arrive at

$$
\dot{\boldsymbol{w}}(t)=\gamma y(t)(\boldsymbol{\xi}(t)-y(t) \boldsymbol{w}(t)),
$$

where $y(t)=\boldsymbol{w}(t)^{T} \boldsymbol{\xi}(t)$.

Note that the biologically plausible learning rule (13) and the adaptation law of the robotic controller (5) are equivalent. Therefore, the neuron model interpretation given above explains the behavior of our cyclic motion controller from the input to the hidden layer neuron including the adaptation of the sensory synapses. Nevertheless, as can be seen also from Fig. 3, the proposed control law in its current form requires the weights of the input network to be "copied" to the output network. This is in contradiction to the generally accepted principle of local weight adaptation. However, the papers [32], [33] already provide a hint on how the mechanism of "copying" synaptic weights might be implemented in biology. Extending the network structure to address this problem is topic of our current work.

\section{Simulation}

The performance of the cyclic motion controller is validated in simulation for the compliantly actuated quadruped robot depicted in Fig. 4(a). The considered quadruped has four legs and a total number of 12 hinge joints (two

\footnotetext{
${ }^{3}$ Note that thereby Hebb's postulate of locality is still preserved.
}

perpendicular hinge joints in each hip and one in each knee), i.e. $n=12$. The joints of each leg are actuated via linear springs $\partial U_{\psi}(\boldsymbol{x}) / \partial \boldsymbol{q}_{i}=\boldsymbol{K}_{i}\left(\boldsymbol{q}_{i}-\boldsymbol{\theta}_{i}\right)$ with stiffness matrices $\boldsymbol{K}_{i}=\operatorname{diag}(30,7.5,9) \mathrm{Nm} / \mathrm{rad}$. Linear, viscous damping produces torques $\boldsymbol{d}_{i}=\boldsymbol{D}_{i}\left(\dot{\boldsymbol{q}}_{i}-\dot{\boldsymbol{\theta}}_{i}\right)$ with $\boldsymbol{D}_{i}=$ $\operatorname{diag}(0.6,0.075,0.075) \mathrm{Nms} / \mathrm{rad}$ in the joints. The mass distribution of the trunk and legs are given in Fig. 4(a). Furthermore, ground contact points are considered at the tips of each leg. Thereby, a Coulomb friction constant of $\mu=$ 0.75 has been assumed. The controller implementation given in the Appendix I is applied. The parameters of the switching controller have been chosen $\epsilon_{\varphi_{z}}=0.2 \mathrm{rad}$ and $\hat{\theta}_{z}=1.0 \mathrm{rad}$. The initial weights of the adaptation controller have been chosen such that in the initial configuration (Fig. 4(a)) a generalized modal deflection would produce a pure vertical force at the tips of the legs (repelling the quadruped from the ground).

A vertical jumping motion is simulated. To demonstrate the adaptation properties of the controller, the mechanical joint stiffness of the robot is changed during the experiment. At $t \approx 6 \mathrm{sec}$. (when the potential energy of the springs is approximately zero) the joint stiffness is increased instantaneously to $1.5 \boldsymbol{K}_{i}$. Fig. 4(b) depicts the phase plot of the generalized modal motion. It can be seen that the motion initially converges to a limit cycle corresponding to the initial stiffness (blue line) and then converges to a different limit cycle due to the increased stiffness (red line). The corresponding time-plots of the vertical trunk motion and the link positions of one of the legs are shown in Fig. 4(c) and Fig. 4(d), respectively. Herein, it can be observed that as a result of the increased stiffness, the controller adapts to the decreased frequency of the oscillation mode (the frequency decreases since the jumping height and therefore the duration of the flight-phase increases). Moreover, Fig. 4(e) depicts the motion of the first three modal coordinates. Note that in the steady-state phase of the oscillation, mainly the motion of the most dominant oscillation mode is excited, while the motion along the other modes converges almost to zero. A video exemplifying the simulation is appended to this paper. In summary, this experiment clearly demonstrates the advantageous adaptation properties of our novel cyclic motion control concept.

\section{CONCLUSION AND DISCUSSION}

The main contribution of the paper is a biologically plausible hypothesis for neural feedback control of full body resonance limit cycles in biological organisms. This hypothesis might provide an explanation for the easiness and robustness with which animals can swim, crawl, run or fly and thereby adapt to changes in body or environment properties.

Nevertheless, it has to be pointed out that the concept has still open questions which need to be further addressed, possibly in close cooperation with neuro-scientists. The most obvious open question is the current requirement to "copy" the weights adapted in the sensory synapses to the output synapses. Several local adaptation alternatives could 
mass: $4 \mathrm{~kg}$

inertia: $\operatorname{diag}(0.0167,0.0567,0.0667) \mathrm{kgm}^{2}$

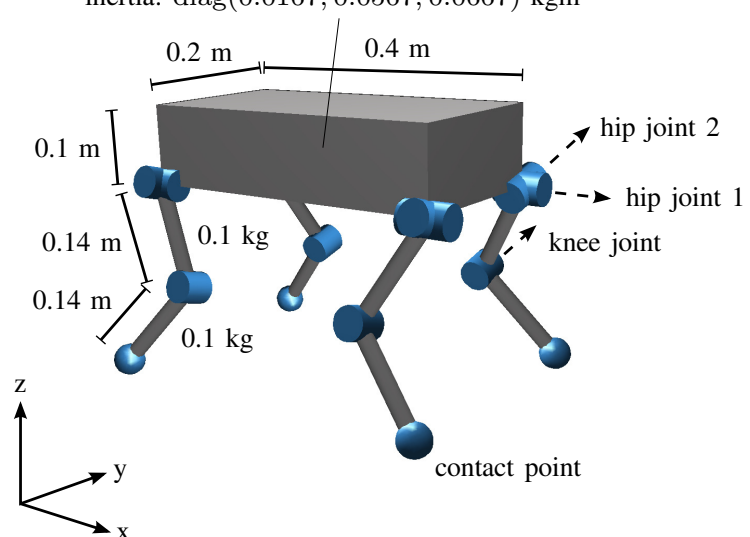

(a) Simulated quadruped. The center of masses of all bodies are placed at the volume center of either the cuboid or grey cylinders.

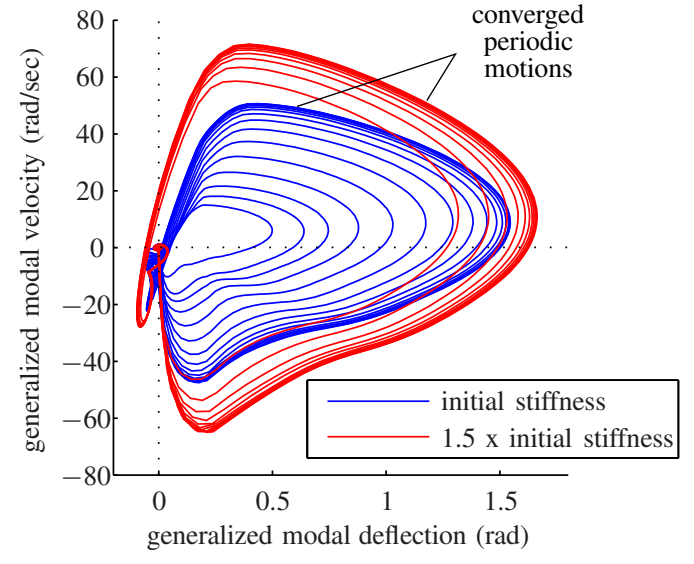

(b) Phase plot: generalized modal deflection vs. velocity.

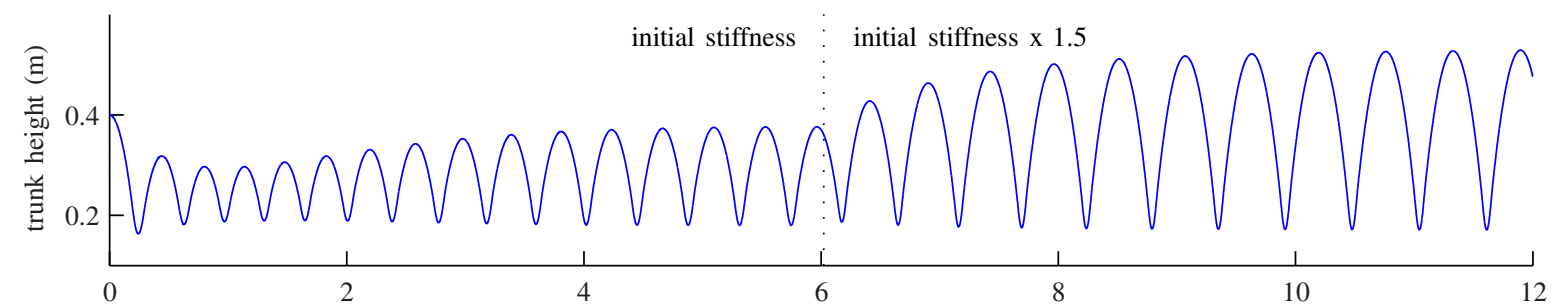

(c) Vertical motion of the trunk.

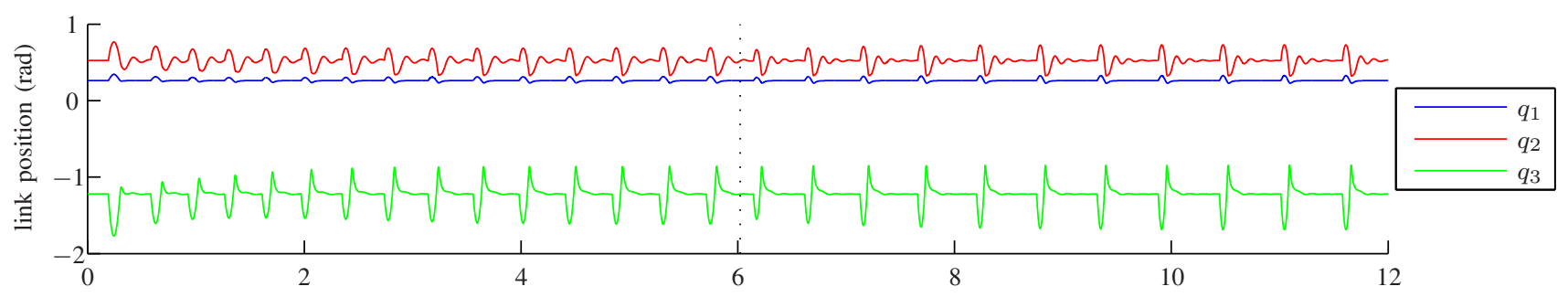

(d) Motion of the link coordinates of one of the legs.

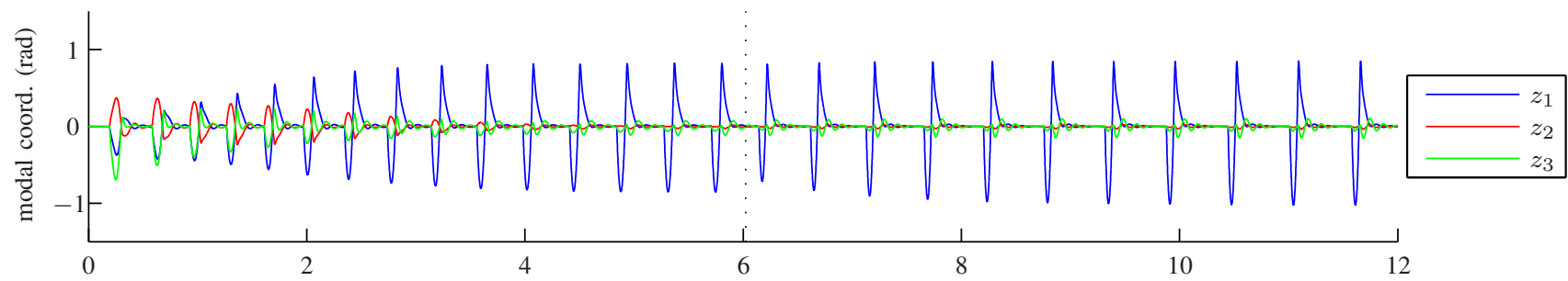

(e) Motion of the first three modal coordinates corresponding to the major principal components.

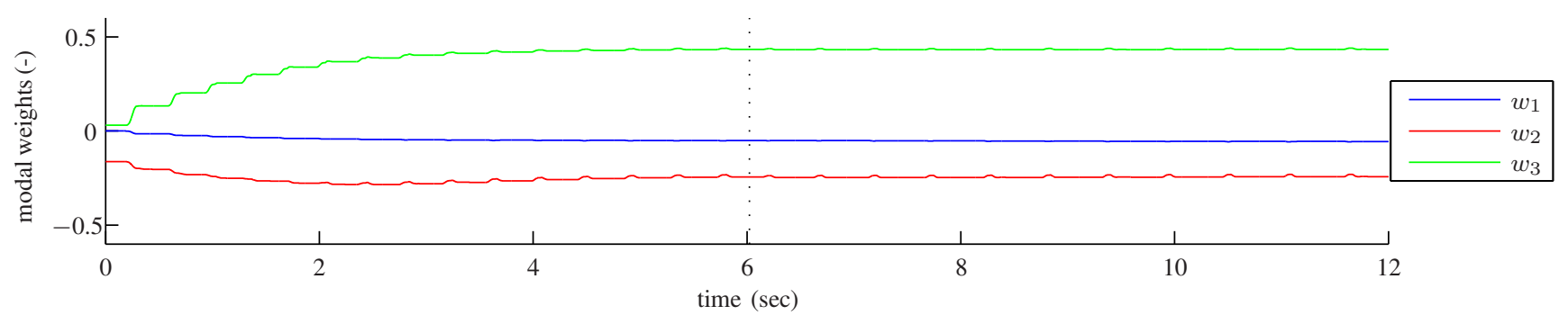

(f) Modal weights corresponding to the actuated joints of one of the legs.

Fig. 4. Simulation results for the quadruped robot shown in (a). At $t \approx 6 \mathrm{sec}$. the mechanical stiffness of the robot is increased. 
be drafted. They need however to be discussed and validated based on the knowledge of existing biological neural circuits in future work. It is our hope that this paper will inspire biologist to experimentally verify the presented hypothesis.

\section{APPENDIX I \\ GENERAL CONTROLLER FORMULATION}

Here, we provide the general controller formulation. Therefore, consider an arbitrary center of oscillation in the motor position $\boldsymbol{\theta}_{0} \in \mathbb{R}^{m}$. Regarding boundedness conditions for the total potential energy $U(\boldsymbol{x})$ detailed in [25], we can compute link positions $\overline{\boldsymbol{q}}\left(\boldsymbol{\theta}_{0}\right)$ satisfying the equilibrium condition

$$
\partial U(\boldsymbol{\theta}, \boldsymbol{q}) / \partial \boldsymbol{q}\left(\boldsymbol{\theta}=\boldsymbol{\theta}_{0}, \boldsymbol{q}=\overline{\boldsymbol{q}}\left(\boldsymbol{\theta}_{0}\right)\right)-\boldsymbol{\tau}_{\mathrm{ext}}=\mathbf{0} .
$$

Using, the static equilibrium position $\overline{\boldsymbol{q}}\left(\boldsymbol{\theta}_{0}\right)$ the generalized spring deflection takes the form

$$
\Delta \varphi_{z}=\boldsymbol{w}^{T}\left\{\boldsymbol{\theta}-\boldsymbol{q}-\left[\boldsymbol{\theta}_{0}-\overline{\boldsymbol{q}}\left(\boldsymbol{\theta}_{0}\right)\right]\right\},
$$

such that (3) changes to

$$
\Delta \theta_{z}\left(\varphi_{z}\right)=\left\{\begin{array}{cc}
\operatorname{sign}\left(\Delta \varphi_{z}\right) \hat{\theta}_{z} & \text { if }\left|\Delta \varphi_{z}\right|>\epsilon_{\varphi_{z}} \\
0 & \text { otherwise }
\end{array}\right.
$$

Moreover, the link side motion w.r.t. the static equilibrium position $\overline{\boldsymbol{q}}\left(\boldsymbol{\theta}_{0}\right)$ is considered in the adaptation law (5):

$$
\dot{\boldsymbol{w}}(t)=\gamma z(t)\left[\left(\boldsymbol{q}(t)-\overline{\boldsymbol{q}}\left(\boldsymbol{\theta}_{0}\right)\right)-z(t) \boldsymbol{w}(t)\right] .
$$

Herein, $z(t)=\boldsymbol{w}(t)^{T}\left(\boldsymbol{q}(t)-\overline{\boldsymbol{q}}\left(\boldsymbol{\theta}_{0}\right)\right)$. Finally, the controller output takes the form

$$
\boldsymbol{\theta}=\boldsymbol{\theta}_{0}+\boldsymbol{w}(t) \Delta \varphi_{z}
$$

\section{APPENDIX II}

\section{INTERMEDIATE COMPUTATION STEP FROM (11) TO (12)}

We make use of the binomial series $(1+x)^{-1 / 2}=1-$ $1 / 2 x+3 / 8 x^{2}-5 / 16 x^{3} \ldots$ and keep only terms up to $\mathcal{O}(\gamma)$ :

$$
\begin{gathered}
\|\boldsymbol{w}+\Delta \tilde{\boldsymbol{w}}\|^{-1}=\left(\boldsymbol{w}^{T} \boldsymbol{w}+2 \boldsymbol{w}^{T} \Delta \tilde{\boldsymbol{w}}+\Delta \tilde{\boldsymbol{w}}^{T} \Delta \tilde{\boldsymbol{w}}\right)^{-1 / 2} \\
=\left(1+2 \boldsymbol{w}^{T} \Delta \tilde{\boldsymbol{w}}+\mathcal{O}\left(\gamma^{2}\right)\right)^{-1 / 2}=1-\boldsymbol{w}^{T} \Delta \tilde{\boldsymbol{w}}+\mathcal{O}\left(\gamma^{2}\right) . \\
\text { ACKNOWLEDGMENT }
\end{gathered}
$$

This work has been partially funded by the European Commission's Seventh Framework Programme as part of the project SAPHARI (Grant no. 287513).

\section{REFERENCES}

[1] M. Grebenstein and P. v. d. Smagt, "Antagonism for a highly anthropomorphic hand-arm system," Advanced Robotics, vol. 22, no. 1, pp. $39-55,2008$

[2] S. Wolf and G. Hirzinger, "A new variable stiffness design: Matching requirements of the next robot generation," in IEEE Int. Conf. on Robotics and Automation, 2008.

[3] A. Albu-Schäffer, O. Eiberger, M. Fuchs, M. Grebenstein, S. Haddadin, C. Ott, A. Stemmer, T. Wimböck, S. Wolf, C. Borst, and G. Hirzinger, "Anthropomorphic soft robotics - from torque control to variable intrinsic compliance," in Robotics Research, ser. Springer Tracts in Advanced Robotics, C. Pradalier, R. Siegwart, and G. Hirzinger, Eds. Springer, 2011, vol. 70, pp. 185-207.

[4] M. Grebenstein, A. Albu-Schäffer, T. Bahls, M. Chalon, O. Eiberger, W. Friedl, R. Gruber, S. Haddadin, U. Hagn, R. Haslinger, H. Höppner, S. Jörg, M. Nickl, A. Nothhelfer, F. Petit, J. Reill, N. Seitz, T. Wimböck, S. Wolf, T. Wüsthoff, and G. Hirzinger, "The DLR Hand Arm System," in Proc. IEEE Int. Conf. on Robotics and Automation, 2011.
[5] D. Braun, M. Howard, and S. Vijayakumar, "Exploiting variable stiffness in explosive movement tasks," in Robotics: Science and Systems, 2011.

[6] D. Braun, F. Petit, F. Huber, S. Haddadin, P. van der Smagt, A. AlbuSchaffer, and S. Vijayakumar, "Optimal torque and stiffness control in compliantly actuated robots," in Proc. IEEE/RSJ Int. Conf. on Intelligent Robots and Systems. IEEE, 2012.

[7] S. Haddadin, F. Huber, and A. Albu-Schäffer, "Optimal control for exploiting the natural dynamics of variable stiffness robots," in IEEE Int. Conf. on Robotics and Automation, 2012.

[8] M. Hutter, C. Gehring, M. Bloesch, M. Hoepflinger, C. D. Remy, and R. Siegwart, "Starleth: A compliant quadrupedal robot for fast, efficient, and versatile locomotion," in Int. Conf. on Climbing and Walking Robots (CLAWAR), 2012.

[9] A. Sproewitz, A. Tuleu, M. Vespignani, M. Ajallooeian, E. Badri, and A. Ijspeert, "Towards Dynamic Trot Gait Locomotion-Design, Control and Experiments with Cheetah-cub, a Compliant Quadruped Robot," International Journal of Robotics Research, vol. 32, no. 8, pp. 932-950, 2013

[10] J. M. Winters and L. Stark, "Analysis of fundamental human movement patterns through the use of in-depth antagonistic muscle models," IEEE Transaction on Biomedical Engineering, vol. 32, pp. 826-839, 1985.

[11] model complexity," Biological Cybernetics, vol. 55, pp. 403-420, 1987.

[12] H. Geyer, A. Seyfarth, and R. Blickhan, "Compliant leg behaviour explains basic dynamics of walking and running," Proceedings of the Royal Society B: Biological Sciences, vol. 273, no. 1603, pp. 28612867, 2006.

[13] H.-M. Maus, J. Rummel, and A. Seyfarth, "Stable upright walking and running using a simple pendulum based control scheme," in Advances in Mobile Robotics: Proc. 11th Int. Conf. Climbing and Walking Robots. Coimbra, Portugal: World Scientific, 2008, pp. 623-629.

[14] I. Poulakakis and J. W. Grizzle, "The spring loaded inverted pendulum as the hybrid zero dynamics of an asymmetric hopper," IEEE Transactions on Automatic Control, vol. 54, no. 8, pp. 1779-1793, 2009

[15] G. Garofalo, C. Ott, and A. Albu-Schaffer, "Walking control of fully actuated robots based on the bipedal slip model," in Proc. IEEE Int. Conf. on Robotics and Automation, 2012, pp. 1456-1463.

[16] R. Full and D. Koditschek, "Templates and anchors: neuromechanical hypotheses of legged locomotion on land," J. Exp. Biol., vol. 202, no. 15 , pp. 3325-3332, 1999.

[17] A. Ijspeert, "A connectionist central pattern generator for the aquatic and terrestrial gaits of a simulated salamander," Biol. Cybern., vol. 84 pp. 331-348, 2001.

[18] J. Buchli and A. Ijspeert, "Self-organized adaptive legged locomotion in a compliant quadruped robot," Autonomous Robots, vol. 25, no. 4, pp. 331-347, 2008.

[19] D. Lakatos, F. Petit, and A. Albu-Schäffer, "Nonlinear oscillations for cyclic movements in variable impedance actuated robotic arms," in Proc. IEEE Int. Conf. on Robotics and Automation, 2013.

[20] D. Lakatos, M. Görner, F. Petit, A. Dietrich, and A. Albu-Schäffer, "A modally adaptive control for multi-contact cyclic motions in compliantly actuated robotic systems," in Proc. IEEE/RSJ Int. Conf. on Intelligent Robots and Systems, 2013, pp. 5388-5395.

[21] D. Lakatos, F. Petit, and A. Albu-Schäffer, "Nonlinear oscillations for cyclic movements in human and robotic arms."

[22] W. Gerstner and W. M. Kistler, Spiking neuron models: Single neurons, populations, plasticity. Cambridge university press, 2002.

[23] D. Hebb, The Organisation of Behavior. New York: Wiley, 1949

[24] K. D. Miller and D. J. MacKay, "The role of constraints in hebbian learning," Neural Computation, vol. 6, no. 1, pp. 100-126, 1994

[25] A. Albu-Schäffer, C. Ott, and F. Petit, "Constructive energie shaping control for a class of euler-lagrange systems," in 10th Int. IFAC Symposium on Robot Control, 2012.

[26] P. Kokotovic, H. Khalil, and J. O'Reilly, Singular Perturbation Methods in Control: Analysis and Design. Academic Press, London, 1986.

[27] E. Oja, Subspace methods of pattern recognition. Letchworth England: Research Studies Press and John Wiley and Sons, 1983.

[28] B. Feeny and R. Kappagantu, "On the physical interpretation of proper orthogonal modes in vibrations," Journal of Sound and Vibration, vol. 211, pp. 607-616, 1998

[29] E. Bizzi, N. Hogan, F. A. Mussa-Ivaldi, and S. Giszter, "Does the nervous system use equilibrium-point control to guide single and multiple joint movements?" Behavioral and brain sciences, vol. 15 no. 04, pp. 603-613, 1992

[30] P. Dayan and L. F. Abbott, Theoretical neuroscience. MIT press Cambridge, MA, 2001, vol. 31.

[31] E. Oja, "Simplified neuron model as a principal component analyzer," Journal of mathematical biology, vol. 15, no. 3, pp. 267-273, 1982

[32] C. Fernando, K. Karishma, and E. Szathmáry, "Copying and evolution of neuronal topology," PloS one, vol. 3, no. 11, p. e3775, 2008.

[33] C. Fernando, R. Goldstein, and E. Szathmáry, "The neuronal replicator hypothesis," Neural Computation, vol. 22, no. 11, pp. 2809-2857, 2010. 